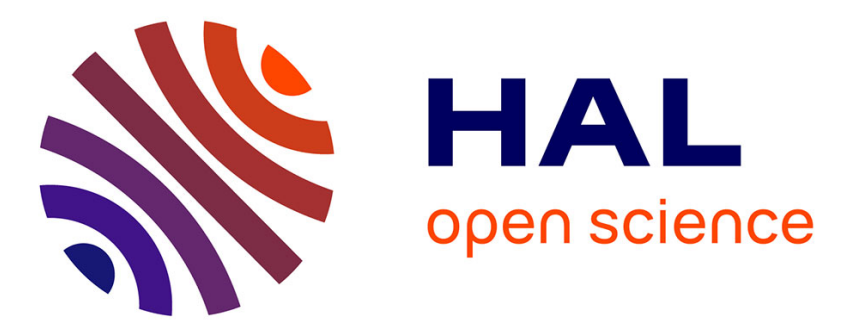

\title{
Generalized Rayleigh criterion for non-axisymmetric centrifugal instabilities
}

\author{
Paul Billant, Francois Gallaire
}

\section{To cite this version:}

Paul Billant, Francois Gallaire. Generalized Rayleigh criterion for non-axisymmetric centrifugal instabilities. Journal of Fluid Mechanics, 2005, 542 (november), pp.365-379. 10.1017/S0022112005006464 . hal-00015707

\section{HAL Id: hal-00015707 https://hal.science/hal-00015707}

Submitted on 16 Jul 2014

HAL is a multi-disciplinary open access archive for the deposit and dissemination of scientific research documents, whether they are published or not. The documents may come from teaching and research institutions in France or abroad, or from public or private research centers.
L'archive ouverte pluridisciplinaire HAL, est destinée au dépôt et à la diffusion de documents scientifiques de niveau recherche, publiés ou non, émanant des établissements d'enseignement et de recherche français ou étrangers, des laboratoires publics ou privés. 


\title{
Generalized Rayleigh criterion for non-axisymmetric centrifugal instabilities
}

\author{
By PAUL BILLANT ${ }^{1}$ AND FRANÇOIS GALLAIRE ${ }^{2}$ \\ ${ }^{1}$ LadHyX, CNRS, École Polytechnique, F-91128 Palaiseau Cedex, France \\ ${ }^{2}$ Laboratoire J.-A. Dieudonné, CNRS, Université de Nice, Sophia Antipolis Parc Valrose, \\ F-06108 Nice, France
}

(Received 17 February 2005 and in revised form 4 July 2005)

The well-known Rayleigh criterion is a necessary and sufficient condition for inviscid centrifugal instability of axisymmetric perturbations. We have generalized this criterion to disturbances of any azimuthal wavenumber $m$ by means of large-axialwavenumber WKB asymptotics. A sufficient condition for a free axisymmetric vortex with angular velocity $\Omega(r)$ to be unstable to a three-dimensional perturbation of azimuthal wavenumber $m$ is that the real part of the growth rate

$$
\sigma(r)=-\mathrm{i} m \Omega(r)+\sqrt{-\phi(r)}
$$

is positive at the complex radius $r=r_{0}$ where $\partial \sigma(r) / \partial r=0$, i.e.

$$
\phi^{\prime}\left(r_{0}\right)=-2 \mathrm{i} m \Omega^{\prime}\left(r_{0}\right) \sqrt{-\phi\left(r_{0}\right)},
$$

where $\phi=\left(1 / r^{3}\right) \partial r^{4} \Omega^{2} / \partial r$ is the Rayleigh discriminant, provided that some a posteriori checks are satisfied. The application of this new criterion to various classes of vortex profiles shows that the growth rate of non-axisymmetric disturbances decreases as $m$ increases until a cutoff is reached. The criterion is in excellent agreement with numerical stability analyses of the Carton \& McWilliams (1989) vortices and allows one to analyse the competition between the centrifugal instability and the shear instability. The generalized criterion is also valid for a vertical vortex in a stably stratified and rotating fluid, except that $\phi$ becomes $\phi=\left(1 / r^{3}\right) \partial r^{4}\left(\Omega+\Omega_{b}\right)^{2} / \partial r$, where $\Omega_{b}$ is the background rotation about the vertical axis. The stratification is found to have no effect. For the Taylor-Couette flow between two coaxial cylinders, the same criterion applies except that $r_{0}$ is real and equal to the inner cylinder radius. In sharp contrast, the maximum growth rate of non-axisymmetric disturbances is then independent of $m$.

\section{Introduction}

In rotating flows, the equilibrium between the centrifugal force and the radial pressure gradient can be unstable and may give rise to centrifugal instability. By an energy argument, Rayleigh (1917) has given a necessary condition for the centrifugal instability in inviscid fluids, which was strengthened by Synge (1933) to a sufficient condition: an axisymmetric flow is unstable with respect to axisymmetric three-dimensional perturbations when the square of the circulation decreases with the radius in some region of the flow. By means of large-axial-wavenumber asymptotics, Bayly (1988) has extended the instability condition to general two-dimensional flows: it is sufficient that the magnitude of the circulation on closed streamlines decreases locally outward. 
However, the Rayleigh criterion only applies to axisymmetric disturbances (or to disturbances which do not vary along streamlines in the case of the generalized version of Bayly 1988). No theoretical result exists on non-axisymmetric centrifugal instabilities in inviscid fluids. As pointed out by Howard \& Gupta (1962) and Howard (1962) (see also Drazin \& Reid 1981, §15.1), the attempt by Chandrasekhar (1961, $\S 67)$ to show that the necessary and sufficient Rayleigh criterion for axisymmetric perturbations also applies to non-axisymmetric disturbances is incorrect. For example, Smyth \& McWilliams (1998) and Gallaire \& Chomaz (2003) have investigated numerically the stability of the Carton \& McWilliams (1989) vortex profiles and shown that only the first azimuthal modes are unstable with growth rates smaller than for the axisymmetric mode. Regarding the Couette flow in the inviscid limit, Krueger \& DiPrima (1962) and Bisshopp (1963) have shown, in some limiting cases (narrow gap, small azimuthal wavenumber), that the axisymmetric mode is always more unstable than non-axisymmetric modes for a given axial wavenumber.

The goal of the present paper is to extend the Rayleigh criterion to nonaxisymmetric perturbations following an approach similar to the recent analysis of Kelvin waves by Le Dizès \& Lacaze (2005). Unstable eigenmodes are constructed for large axial wavenumber using the WKB method (Bender \& Orszag 1978) analogously to the methods used to find approximate solutions of Schrödinger's equation in quantum mechanics.

\section{Stability equations}

We consider a basic flow with velocity components $\left[0, U_{\theta}(r), 0\right]$ in cylindrical coordinates $(r, \theta, z)$ in an inviscid and incompressible fluid. We subject this basic flow to infinitesimal three-dimensional velocity perturbations $\boldsymbol{u}^{\prime}$ written in the form

$$
\boldsymbol{u}^{\prime}(r, \theta, z, t)=\left[u_{r}(r), u_{\theta}(r), u_{z}(r)\right] \mathrm{e}^{\sigma t+\mathrm{i} k z+\mathrm{i} m \theta}+\text { c.c. }
$$

where $\sigma$ is the complex growth rate, $k$ the axial wavenumber, $m$ the azimuthal wavenumber and c.c. denotes the complex conjugate. Defining $\psi=u_{r} \sqrt{r / Q}$ where $Q=k^{2}+\left(m^{2} / r^{2}\right)$, the linearized equations of motion for these perturbations can be reduced to a single equation for $\psi$ (Drazin \& Reid 1981)

with

$$
\frac{\mathrm{d}^{2} \psi}{\mathrm{d} r^{2}}=B \psi
$$

$$
B=-\frac{m^{2}\left(m^{2}+4 k^{2} r^{2}\right)}{r^{2}\left(r^{2} k^{2}+m^{2}\right)^{2}}+\frac{3}{4 r^{2}}+\frac{m^{2}}{r^{2}}+k^{2} \frac{S^{2}+\phi}{S^{2}}+\frac{\mathrm{i} m}{r^{2} S}\left(r \zeta^{\prime}-2 \zeta \frac{k^{2} r^{2}}{m^{2}+k^{2} r^{2}}\right),
$$

where $\Omega=U_{\theta} / r$ is the angular velocity of the basic state, $\zeta=(1 / r) \partial r U_{\theta} / \partial r$ its axial vorticity, $\phi=2 \Omega \zeta$ the Rayleigh discriminant and $S=\sigma+\mathrm{im} \Omega$. In most of the paper, we consider a free vortex in an infinite medium so that the boundary conditions are $u_{r} \rightarrow 0$ as $r \rightarrow \infty$ and, at $r=0, u_{r}=0$ if $|m| \neq 1$ or $\partial u_{r} / \partial r=0$ if $|m|=1$. This implies the boundary conditions $\psi(0)=0$ and $\psi \rightarrow 0$ as $r \rightarrow \infty$ whatever $m$. The particular case of the Couette flow between two coaxial cylinders will be treated in $\S 7$.

\section{WKB stability analysis for large axial wavenumber}

The present analysis stems from the fact that, for large axial wavenumber $k$, the function $B$ is of the form

$$
B(r)=k^{2} B_{0}(r)+O(1) \quad \text { with } \quad B_{0}=1+\frac{\phi}{S^{2}} .
$$


This behaviour allows one to use a WKB approach to find approximate solutions of (2.2). We stress that only unstable eigenmodes $(\operatorname{Re}(\sigma)>0)$ will be considered so that no singularity of $B_{0}$ where $S=0$ exists for real radius. For clarity, we first present the case of axisymmetric modes. Non-axisymmetric modes will be considered in $\S 3.2$.

\subsection{Axisymmetric perturbations}

The case of axisymmetric modes is simpler because the function $B_{0}(r)$ is real for real radius and growth rate. According to WKB theory (see for example, Bender \& Orszag 1978, §10.5; Le Dizès \& Lacaze 2005), it is possible to construct a localized eigenmode satisfying the boundary conditions if we assume that there exist two turning points at $r_{1}$ and $r_{2}$ (with the convention $r_{1}<r_{2}$ ) where $B_{0}\left(r_{1}\right)=B_{0}\left(r_{2}\right)=0$ enclosing a region where $B_{0}$ is negative. For unstable modes (i.e. $\left.\operatorname{Re}(\sigma)>0\right)$ to exist, this requires that $\phi$ reaches a negative minimum between $r_{1}$ and $r_{2}$. In this interval, the WKB approximations of the solution of (2.2) are oscillatory. Outside this region, for $r<r_{1}$ and $r>r_{2}, B_{0}$ is positive so that the two WKB approximations are exponentially growing or decaying. For $0<r<r_{1}$, we retain only the WKB approximation which decays as $r \rightarrow 0$ :

$$
\psi_{1}(r)=\frac{C}{\left(B_{0}(r)\right)^{1 / 4}} \exp \left(-k \int_{r}^{r_{1}} \sqrt{B_{0}(u)} \mathrm{d} u+O\left(\frac{1}{k}\right)\right),
$$

where $C$ is a constant. When $r_{1}$ is not too close to the vortex axis (i.e. $r_{1} \gg 1 / k$ ), Le Dizès \& Lacaze (2005) have shown that this approximation can be matched to Bessel functions which are valid in the axis neighbourhood for $r k=O(1)$ and ensure the boundary condition at $r=0$. At the turning point $r_{1}$, the WKB approximation breaks down and should be replaced by Airy functions. For $r_{1}<r<r_{2}$, the WKB approximation of the solution of (2.2) which matches the approximation for $r<r_{1}$ through the Airy functions at the turning point is

$$
\psi_{2}=\frac{2 C}{\left(-B_{0}(r)\right)^{1 / 4}} \sin \left(k \int_{r_{1}}^{r} \sqrt{-B_{0}(u)} \mathrm{d} u+\frac{\pi}{4}\right),
$$

where classical connection formulae have been used (Bender \& Orszag 1978). Note that the sine factor in (3.3) also neglects $O(1 / k)$ terms, but the $O(1 / k)$ symbol will be omitted from now on for brevity. For $r>r_{2}$, we select the approximation which decays as $r \rightarrow \infty$ :

$$
\psi_{3}=\frac{D}{\left(B_{0}(r)\right)^{1 / 4}} \exp \left(-k \int_{r_{2}}^{r} \sqrt{B_{0}(u)} \mathrm{d} u\right)
$$

where $D$ is a constant. Using connection formulae through the turning point $r_{2}$, this function matches a WKB approximation for $r_{1}<r<r_{2}$ of the form

$$
\psi_{2}^{\prime}=\frac{2 D}{\left(-B_{0}(r)\right)^{1 / 4}} \sin \left(k \int_{r}^{r_{2}} \sqrt{-B_{0}(u)} \mathrm{d} u+\frac{\pi}{4}\right) .
$$

The last condition is that $\psi_{2}$ and $\psi_{2}^{\prime}$ should be identical in the interval $r_{1}<r<r_{2}$, which implies that $D=|C|$ and that a quantization condition

$$
k \int_{r_{1}}^{r_{2}} \sqrt{-B_{0}(u)} \mathrm{d} u=n \pi+\frac{\pi}{2}+O\left(\frac{1}{k}\right),
$$

is satisfied, where $n$ is a non-negative integer which corresponds to the number of oscillations between the two turning points. Since $k \gg 1$, this quantization condition can be satisfied for $n=O(1)$ only if the two turning points $r_{1}$ and $r_{2}$ are very close and 
separated by a distance $r_{2}-r_{1}=O(1 / \sqrt{k})$. Therefore, it is legitimate to approximate $B_{0}$ between the two turning points by its Taylor expansion around the radius $r^{*}$ where $B_{0}$ is minimum: $B_{0}(r)=B_{0}\left(r^{*}\right)+B_{0}^{\prime \prime}\left(r^{*}\right)\left(r-r^{*}\right)^{2} / 2+\cdots$. Using this quadratic approximation and (3.1), (3.6) can be integrated and yields directly

$$
\sigma=\sqrt{-\phi\left(r^{*}\right)+(2 n+1)^{2} \frac{\phi^{\prime \prime}\left(r^{*}\right)}{8 k^{2}}}-\frac{2 n+1}{2 \sqrt{2}} \frac{\sqrt{\phi^{\prime \prime}\left(r^{*}\right)}}{k}+O\left(\frac{1}{k^{2}}\right),
$$

where $r^{*}$ is defined by $\phi^{\prime}\left(r^{*}\right)=0$. This expression is strictly valid only to $O\left(1 / k^{2}\right)$ and is therefore non-consistent. However, we shall see below that it proves to be very close to the integral dispersion relation (3.6) and in excellent agreement with the exact results even for $k=O(1)$ for the vortex profiles investigated. The corresponding consistent expression

$$
\sigma=\sqrt{-\phi\left(r^{*}\right)}-\frac{2 n+1}{2 \sqrt{2} k} \sqrt{\phi^{\prime \prime}\left(r^{*}\right)}+O\left(\frac{1}{k^{2}}\right),
$$

is identical to the local result of Bayly (1988) for axisymmetric streamlines. Because $\phi^{\prime \prime}\left(r^{*}\right)>0$ by our assumption that $\phi$ has a negative minimum, the maximum growth rate is obtained when $k \rightarrow \infty$ and for the lowest mode $n=0$. Therefore, a sufficient condition to have unstable axisymmetric eigenmodes is that the minimum of $\phi$ is negative, thereby retrieving the sufficient condition for centrifugal instability associated with the Rayleigh criterion.

In figure 1, the asymptotic dispersion relation (3.6) for the most unstable mode $(n=0)$ is compared to the exact growth rate in the case of the Carton \& McWilliams (1989) vortex profiles:

$$
U_{\theta}=r \exp \left(-r^{\alpha}\right)
$$

for the parameter values $\alpha=2$ and $\alpha=4$. As illustrated in Carnevale \& Kloosterziel (1999) or Gallaire \& Chomaz (2003), these vortices are surrounded by a ring of opposite vorticity making then centrifugally unstable for all $\alpha>0$. A comparison of figures $1(a)$ and $1(b)$ shows that the maximum growth rate of the axisymmetric mode $m=0$ increases with $\alpha$ (the $y$-axis scale differs for the two plots). The exact growth rate has been obtained by solving numerically (2.2) using a Chebyshev pseudospectral collocation method, with the collocation points in the interval $y \in[-1,1]$ being mapped to the interval $r \in\left[0, r_{\max }\right]$ through $r=2(1+y) /\left(1-y+4 / r_{\max }\right)$, where $r_{\max }=10$. The number of collocation points has been set to $N=100$, allowing for convergence. The accuracy of the computation has been checked by an independent shooting method. Figure 1 shows that the integral dispersion relation (3.6) provides extraordinarily accurate results even for small values of $k$ : it is indistinguishable from the exact result. For $\alpha=2$ (figure 1a), we also show the non-consistent and consistent quadratic approximations (3.7) and (3.8). The non-consistent quadratic approximation (3.7) is very close to the growth rate given by the integral dispersion relation (3.6), demonstrating that the quadratic approximation is legitimate. The consistent quadratic approximation (3.8) is also in good agreement at large axial wavenumbers but differs for $k=O(1)$.

\subsection{Non-axisymmetric perturbations}

We now consider the general case where $m \neq 0$. The function $B_{0}(r)$ is then complex when $r$ is real implying that the turning points will be complex. Thus, when constructing an eigenmode valid for real $r$, no turning point will be encountered along the real- $r$ axis so that one might think that either of the two WKB approximations 

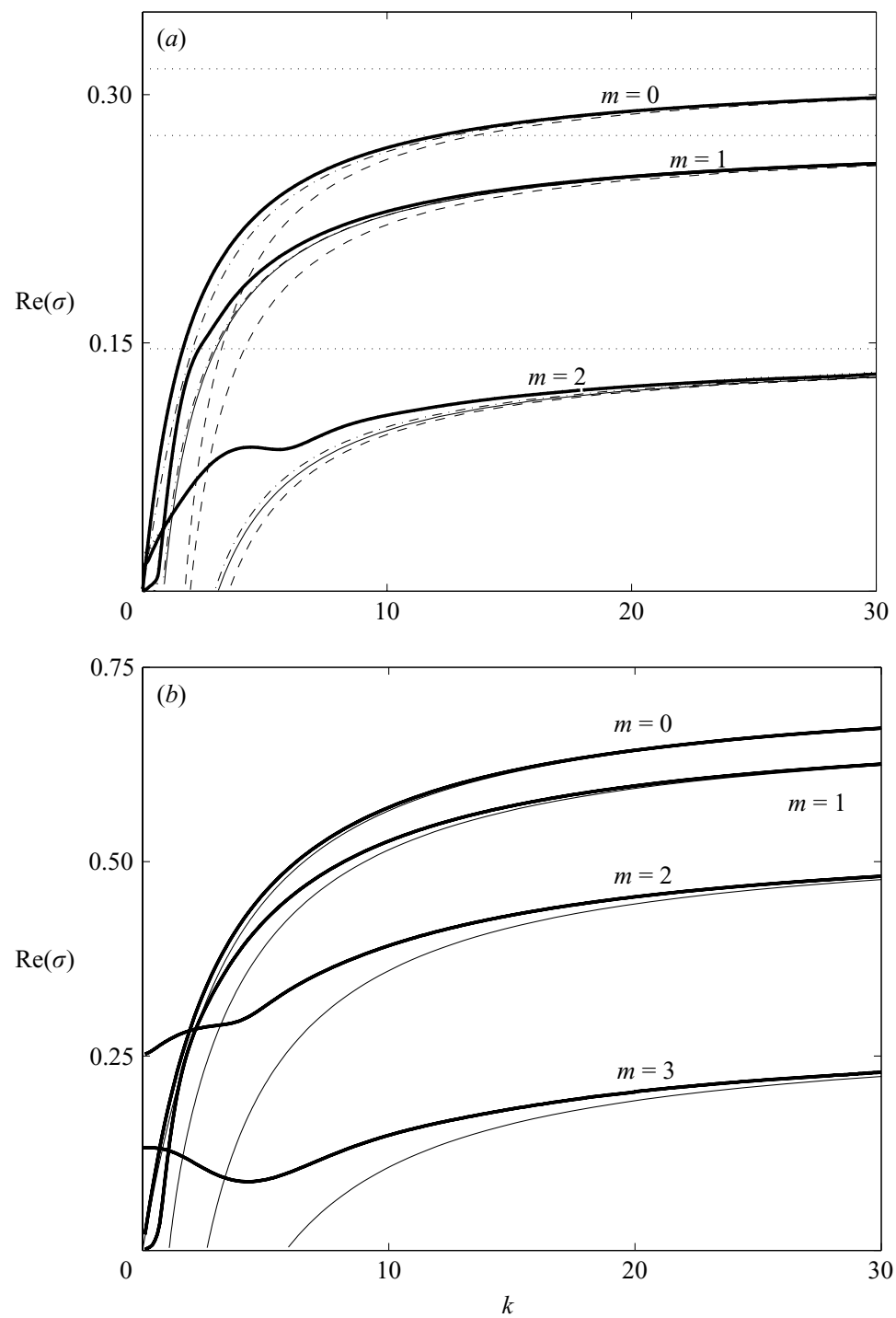

FiguRE 1. Comparison between the asymptotic dispersion relations and the exact growth rates for the Carton \& McWilliams vortex for $(a) \alpha=2$ and $(b) \alpha=4$. For each unstable azimuthal wavenumber, the exact growth rate is shown by a heavy solid line and the asymptotic growth rate given by $(3.15)$ by a thin solid line $(-)$. For $(a)$, the different asymptotic approximations are also shown: $-\cdot-,(3.16) ;---,(3.17 a) ; \cdots,(3.18)$. Note that the asymptotic growth rate for $m=0$ can also be obtained from the relations derived in $\S 3.1:-,(3.6) ;-\cdot-,(3.7)$; ---, (3.8).

of the solution of (2.2),

$$
\psi(r)=\frac{C}{\left(B_{0}(r)\right)^{1 / 4}} \mathrm{e}^{ \pm k \Delta(r)} \quad \text { where } \quad \Delta(r)=\int_{r_{1}}^{r} \sqrt{B_{0}(u)} \mathrm{d} u,
$$

would be valid throughout the whole real axis (the lower bound $r_{1}$ of the integral is chosen to be a complex turning point without loss of generality). However, if there exists a real radius $\tilde{r}_{1}$ such that $\operatorname{Re}\left(\Delta\left(\tilde{r}_{1}\right)\right)=0$, an exponentially growing WKB 


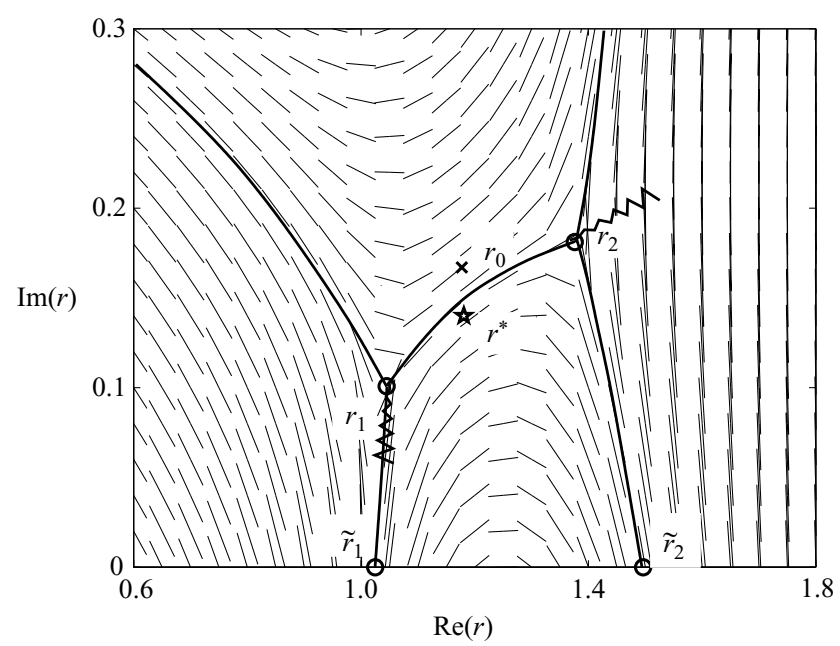

FIGURE 2. Example of the Stokes lines (shown by heavy lines) network for the Carton \& McWilliams vortex for $\alpha=2, m=1$ and $k=10$. The double turning point $r_{0}$ for $k \rightarrow \infty$ is shown by $\times$, the point $r^{*}$ by $\star$ and the two turning points $r_{1}$ and $r_{2}$ by $\bigcirc$. The short lines indicate the direction of the characteristics $\operatorname{Re}(\Delta)=$ const. The branch cuts are represented by wavy lines.

approximation for $r<\tilde{r}_{1}$ becomes exponentially decaying for $r>\tilde{r}_{1}$ and vice versa. This means that, at $\tilde{r}_{1}$, we can switch to the other WKB approximation or to a combination of the two. The equation

$$
\operatorname{Re}(\Delta(r))=0
$$

is the definition of the Stokes lines which delimit the domains in the complex plane where the WKB approximations keep the same behaviour (Bender \& Orszag 1978; Olver 1974). Three Stokes lines emanate from a simple turning point.

In order to construct an eigenmode on the real axis, we shall assume that there exist two complex turning points $r_{1}$ and $r_{2}$ and that for each of them, one emanating Stokes line crosses the real axis at respectively $\tilde{r}_{1}$ and $\tilde{r}_{2}$, as illustrated in figure 2 . Then, the approach closely follows the one for $m=0$. On the real axis, for $0<r<\tilde{r}_{1}$, we select the decaying WKB approximation

$$
\psi_{1}=\frac{C}{\left(B_{0}(r)\right)^{1 / 4}} \exp \left(-k \int_{r}^{r_{1}} \sqrt{B_{0}(u)} \mathrm{d} u\right)
$$

Even if the turning point $r_{1}$ is now complex, the matching WKB approximation for $\tilde{r}_{1}<r<\tilde{r}_{2}$ has the same form as (3.3) (Olver 1974):

$$
\psi_{2}=\frac{2 C}{\left(-B_{0}(r)\right)^{1 / 4}} \sin \left(k \int_{r_{1}}^{r} \sqrt{-B_{0}(u)} \mathrm{d} u+\frac{\pi}{4}\right) .
$$

Finally, the solution is restricted to the decaying WKB approximation for $r>\tilde{r}_{2}$ :

$$
\psi_{3}=\frac{D}{\left(B_{0}(r)\right)^{1 / 4}} \exp \left(-k \int_{r_{2}}^{r} \sqrt{B_{0}(u)} \mathrm{d} u\right) .
$$

The matching between (3.13) and (3.14) at the turning point $r_{2}$ imposes the same quantization condition as for $m=0$,

$$
k \int_{r_{1}}^{r_{2}} \sqrt{-B_{0}(u)} \mathrm{d} u=n \pi+\frac{\pi}{2}+O\left(\frac{1}{k}\right),
$$


except that $\left(r_{1}, r_{2}\right)$ and $B_{0}$ are now complex. A by-product of this condition is $\operatorname{Re}\left(\Delta\left(r_{2}\right)\right)=0$ meaning that the two turning points $r_{1}$ and $r_{2}$ must be connected by a Stokes line (figure 2). As for $m=0, r_{1}$ and $r_{2}$ should be very close for large $k$, allowing a Taylor expansion of $B_{0}$ around the complex radius $r^{*}$ where $B_{0}^{\prime}\left(r^{*}\right)=0$. This yields

$$
k \frac{B_{0}\left(r^{*}\right)}{\sqrt{B_{0}^{\prime \prime}\left(r^{*}\right)}}=-\frac{2 n+1}{\sqrt{2}}+O\left(\frac{1}{k}\right) .
$$

The implicit dispersion relation (3.15) together with $B_{0}\left(r_{1}\right)=B_{0}\left(r_{2}\right)=0$ or (3.16) together with $B_{0}^{\prime}\left(r^{*}\right)=0$ can be solved numerically by an iterative Newton-Raphson method: for a given value of the growth rate $\sigma_{r}$, the frequency $\sigma_{i}$ is determined such that $\operatorname{Im}(k)$ vanishes. Note that the branch cuts of the square root in (3.15) are chosen such that they do not cross the integration path between $r_{1}$ and $r_{2}$ (see figure 2).

However, $\sigma$ and $r^{*}$ can be obtained explicitly in the form

$$
\sigma=\sigma_{0}+\frac{\sigma_{1}}{k}+\cdots, \quad r^{*}=r_{0}+\frac{r_{1}}{k}+\cdots,
$$

where

$$
\begin{aligned}
\sigma_{0} & =-\mathrm{i} m \Omega\left(r_{0}\right)+\sqrt{-\phi\left(r_{0}\right)}, \\
\sigma_{1} & =-\frac{2 n+1}{2 \sqrt{2}} \sqrt{\phi^{\prime \prime}\left(r_{0}\right)-2 m^{2} \Omega^{\prime 2}\left(r_{0}\right)+2 \mathrm{i} m \sqrt{-\phi\left(r_{0}\right)} \Omega^{\prime \prime}\left(r_{0}\right)},
\end{aligned}
$$

and $r_{0}$ is a double turning point where the $r$-derivative of $\sigma_{0}(r) \equiv-\mathrm{i} m \Omega(r)+\sqrt{-\phi(r)}$ vanishes:

$$
\phi^{\prime}\left(r_{0}\right)=-2 \mathrm{i} m \Omega^{\prime}\left(r_{0}\right) \sqrt{-\phi\left(r_{0}\right)} .
$$

This allows us to generalize the Rayleigh criterion to non-axisymmetric disturbances: Generalized RAYLEIGH CRITERION: A sufficient condition for a perturbation with an azimuthal wavenumber $m$ to be unstable is that the real part of the growth rate $\sigma_{0}$ given by (3.18) is positive at the complex radius $r_{0}$ defined by (3.20).

It should be stressed that this criterion is valid only if there exist two Stokes lines which cross the real axis as in figure 2. In addition, the WKB approximations should be uniformly valid on the real axis in each interval. However, as explained by Le Dizès \& Lacaze (2005), the above criterion is expected to remain valid if there exist two contours connecting $r_{0}$ to 0 and $r_{0}$ to $\infty$ on which the WKB approximations are uniformly valid, i.e. $\operatorname{Re}(\Delta)$ is monotonic (Olver 1974). These two conditions should be checked a posteriori. For all the vortex profiles investigated, we have observed that only the double turning point $r_{0}(\mathrm{~m})$ which derives continuously (assuming $m$ real) from the one for $m=0$ satisfies these conditions.

Figure 1(a) compares the different asymptotic growth rates given by (3.15), (3.16) and (3.17a) for $m=1$ and $m=2$ to the exact growth rates for the Carton \& McWilliams vortex (3.9) for $\alpha=2$. The agreement between the three asymptotic formulae and the numerical results is excellent for large axial wavenumber, the integral form (3.15) and the non-consistent quadratic approximation (3.16) being the most accurate. For small $k$, the agreement continues to be satisfactory for $m=1$ whereas, for $m=2$, the asymptotic growth rate goes to zero for $k \approx 3$ while the exact growth rate remains positive at $k=0$. This difference is due to the two-dimensional $(k=0)$ shear instability (see §5) which is not captured by the present analysis strictly valid for $k \gg 1$.

The same trends are seen in figure $1(b)$ for the value $\alpha=4$ : the asymptotic growth rate given by (3.15) is in excellent agreement with the exact growth rates except at 
small axial wavenumber for $m=2$ and $m=3$ because of the two-dimensional shear instability. Figure $1(a, b)$ also shows that the maximum growth rate of each mode decreases with $m$ but increases with $\alpha$. Strikingly, no instability has been found for $m \geqslant 4$ when $\alpha=4$ and for $m \geqslant 3$ when $\alpha=2$, in agreement with Smyth $\&$ McWilliams (1998). These features will be further investigated in the next section.

\section{Analytical results}

The maximum growth rate (3.18) can be determined analytically in the case of the Carton \& McWilliams vortex profiles (3.9) for any value of $\alpha$ :

$$
\sigma_{0}=\frac{1}{2} \exp \left[-\frac{1}{4 \alpha}\left(8+2 \alpha-m^{2}+\mathrm{i} m \sqrt{4 \alpha-m^{2}}\right)\right]\left(\sqrt{4 \alpha-m^{2}}-\mathrm{i} m\right) .
$$

As seen in (4.1) and in figure 3(a), the real part of the growth rate $\sigma_{0 r}$ is positive and maximum for $m=0$ and continuously decreases with $m$ until the cutoff $m_{c}=2 \sqrt{\alpha}$. This azimuthal wavenumber cutoff explains why only the modes $0 \leqslant m \leqslant 2$ were found to be unstable for $\alpha=2$ (figure $1 a$ ) and the modes $0 \leqslant m \leqslant 3$ for $\alpha=4$ (figure $1 b$ ). It is worth emphasizing that this cutoff is not due to a viscous damping, contrary to what was suggested by Gallaire \& Chomaz (2003). It can be checked that $\partial \sigma_{0 r} / \partial m$ is negative for all $m$ and $\alpha$, demonstrating that the axisymmetric mode is always the most unstable. This feature seems generic. For example, the axisymmetric mode is also the most unstable mode for the vortex profiles $U_{\theta}=r /\left(1+r^{b}\right)$ and $U_{\theta}=r /\left(1+r^{2}\right)^{b}$ introduced by Gent \& McWilliams (1986). The cutoff azimuthal wavenumbers are $m_{c}=\sqrt{6(b-2)}$ and $m_{c}=2 \sqrt{(b-1)(1+2 b)} / b$ respectively.

For arbitrary vortex profiles, it is possible to make two general statements regarding the dominance of the axisymmetric mode. First, we deduce from (3.18) and (3.20) that

$$
\begin{gathered}
\frac{\partial \sigma_{0}}{\partial m}=-\mathrm{i} \Omega\left(r_{0}\right), \\
\frac{\partial^{2} \sigma_{0}}{\partial m^{2}}=\frac{2 \Omega^{\prime 2}\left(r_{0}\right) \phi\left(r_{0}\right)}{\phi^{\prime \prime}\left(r_{0}\right) \sqrt{-\phi\left(r_{0}\right)}-\mathrm{i} m\left(\Omega^{\prime \prime}\left(r_{0}\right) \phi\left(r_{0}\right)+\Omega^{\prime}\left(r_{0}\right) \phi^{\prime}\left(r_{0}\right)\right)},
\end{gathered}
$$

using implicit differentiation. Because $\Omega\left(r_{0}\right)$ is real for $m=0$, we have $\partial \sigma_{0 r} / \partial m=0$ for $m=0$. In addition, $\phi^{\prime \prime}\left(r_{0}\right)>0$ for $m=0$ because $r_{0}$ is a minimum of $\phi$, implying that $\partial^{2} \sigma_{0} / \partial m^{2}$ is real and negative. Thus, $m=0$ is a local maximum of the curve $\sigma_{0 r}(m)$.

Second, we show that non-axisymmetric modes cannot be more unstable than the $m=0$ mode by demonstrating that $\sqrt{-\min (\phi)}$ is an upper bound of the growth rate for any azimuthal wavenumber $m$ in the large-axial-wavenumber limit. By multiplying equation $(2.2)$ by the complex conjugate $\psi^{*}$ and by integrating from zero to infinity, we obtain after an integration by parts

$$
-\int_{0}^{\infty}\left|\frac{\mathrm{d} \psi}{\mathrm{d} r}\right|^{2} \mathrm{~d} r=k^{2} \int_{0}^{\infty} B_{0}|\psi|^{2} \mathrm{~d} r
$$

for $k \gg 1$. This shows that a necessary condition for $\psi$ to be non-zero is that the real part of $B_{0}$ be negative for some radius: $\operatorname{Re}\left(B_{0}\right)=1+\phi\left(S_{r}^{2}-S_{i}^{2}\right) /\left(S_{r}^{2}+S_{i}^{2}\right)^{2}<0$, where $S=S_{r}+\mathrm{i} S_{i}$. Since $\operatorname{Re}\left(B_{0}\right)$ is bounded by its value for $S_{i}=0$ when $\phi$ is negative, one has $\left(\phi+S_{r}^{2}\right) / S_{r}^{2}<\operatorname{Re}\left(B_{0}\right)<0$. This directly implies the necessary condition that $S_{r}^{2}<-\min (\phi)$ independently of $m$. 

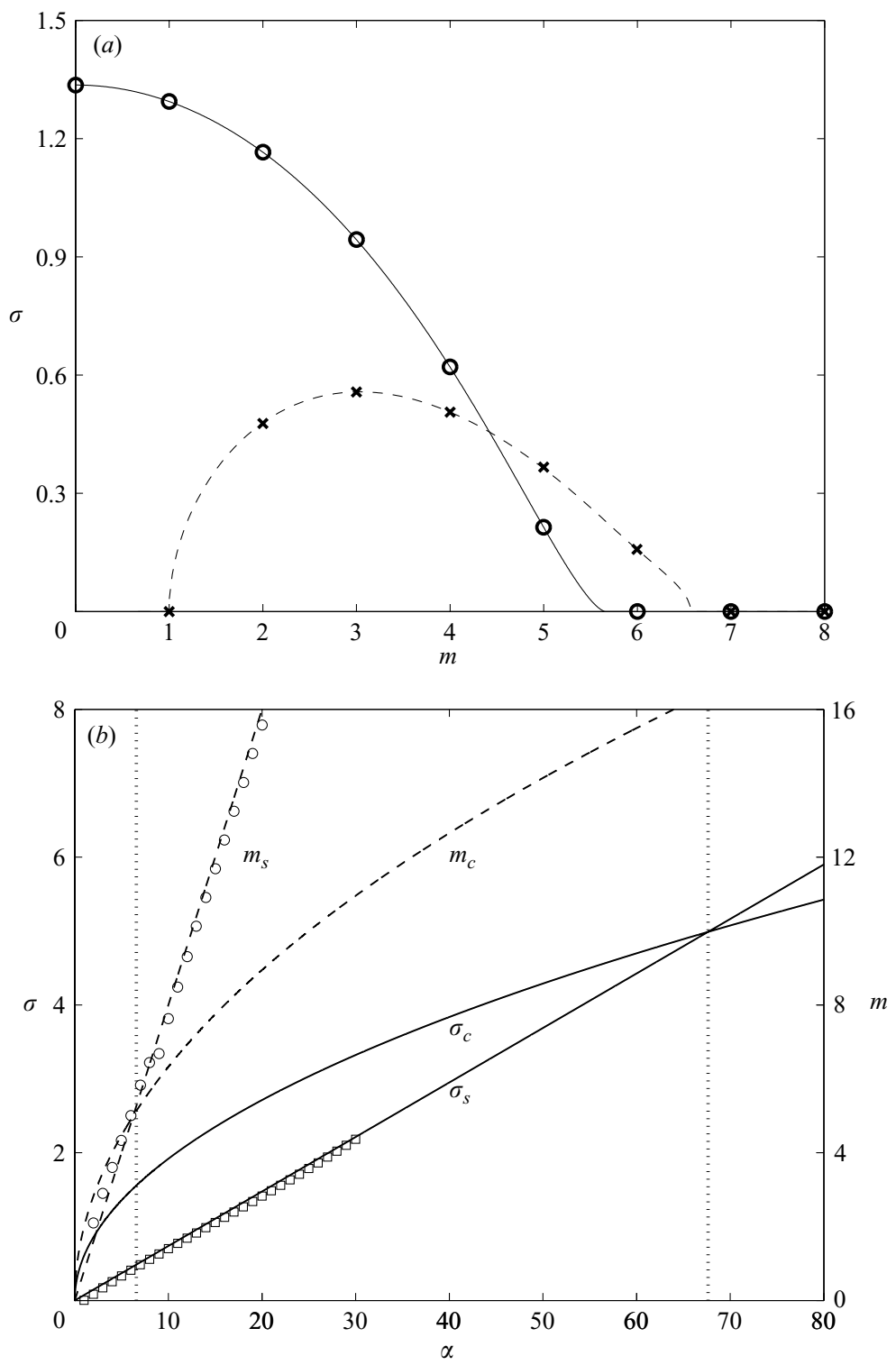

FiguRE 3. (a) Growth rates of the centrifugal instability for $k=\infty$ (solid line) and shear instability for $k=0$ (dashed line) for the Carton \& McWilliams vortices (3.9) for $\alpha=8$. (b) Maximum growth rates (solid lines) and azimuthal cutoff wavenumbers (dashed lines) of the centrifugal and shear instabilities as a function of $\alpha$. The results for $\alpha>30$ for the shear instability (lines) have been obtained by extrapolating the numerical results for $\alpha<30$ (symbols) by a linear fit.

\section{Competition between the centrifugal and shear instabilities}

Besides being unstable to the three-dimensional centrifugal instability, the Carton \& McWilliams vortices (3.9) are also unstable in the two-dimensional limit with respect to the shear instability as seen in figure 1 . The competition between these two different instabilities is illustrated in figure $3(a)$ for $\alpha=8$. For this value of $\alpha$, the centrifugal instability (for $k=\infty$ ) is the most unstable for $m=0$ and occurs in the azimuthal 
wavenumber band $0 \leqslant m \leqslant 5$. The shear instability (for $k=0$ ) exists for $2 \leqslant m \leqslant 6$ and is maximum for $m=3$.

The competition is further analysed as a function of $\alpha$ in figure $3(b)$, where the maximum growth rate $\sigma_{c}(\alpha) \equiv \sigma_{0}$ of the centrifugal instability given by (4.1) for $m=0$ and the cutoff wavenumber $m_{c}(\alpha)=2 \sqrt{\alpha}$ are compared to the maximum growth rate of the shear instability $\sigma_{s}(\alpha)$ and the associated upper cutoff wavenumber $m_{s}(\alpha)$ obtained numerically (note that the lower cutoff remains always equal to $m=1$ ). For the centrifugal instability, both $\sigma_{c} \sim \mathrm{e}^{-1 / 2} \sqrt{\alpha}$ and $m_{c}$ scale like $\sqrt{\alpha}$. In contrast, $\sigma_{s}(\alpha)$ and $m_{s}(\alpha)$ are seen to vary linearly with $\alpha$ in agreement with the results of Carnevale \& Kloosterziel (1994) for $\alpha<10$. This linear dependence can be physically understood by drawing an analogy between a plane shear and the radial shear in circular geometry. It is known from plane shear layer instability theory (see Drazin \& Reid 1981) that both the growth rate and the cutoff wavenumbers scale like the inverse of the shear layer thickness $\delta$. For the Carton \& McWilliams vortex profile, it is easy to show that the shear layer thickness scales like the inverse of $\alpha$, implying $\sigma_{s} \sim \alpha$ and $m_{s} \sim \alpha$. As a result of these scaling laws, the two-dimensional shear instability is expected to be more unstable than the three-dimensional centrifugal instability for large values of $\alpha$. In practice, the prefactors are such that this occurs only for very high values: $\alpha>68$. However, when $\alpha>6.25$, the azimuthal cutoff wavenumber $m_{s}$ of the shear instability is larger than the cutoff $m_{c}$ of the centrifugal instability (figure $3 b$ ).

\section{Further generalization to stably stratified and rotating fluids}

The additional effects due to a background rotation about the vertical axis and a stable stratification under the Boussinesq approximation can be easily taken into account in the generalized Rayleigh criterion in the case of a vertical vortex.

The function $B$ corresponding to (2.3) becomes

$$
B=-\frac{H^{\prime \prime}}{H}+2 \frac{H^{\prime 2}}{H^{2}}+\frac{m^{2}}{r^{2}}+k^{2} \frac{S^{2}+\phi_{g}}{S^{2}+N^{2}}+\frac{\mathrm{i} m}{r^{2} S}\left[r \zeta^{\prime}-2\left(\zeta+2 \Omega_{b}\right)\left(\frac{r Q^{\prime}}{Q}+2\right)\right],
$$

where $H=\sqrt{r Q}, Q=\left(k^{2} S^{2} /\left(S^{2}+N^{2}\right)\right)+m^{2} / r^{2}, N$ is the Brunt-Väisälä frequency, $\phi_{g}=\left(2 \Omega+2 \Omega_{b}\right)\left(\zeta+2 \Omega_{b}\right)$ is the generalized Rayleigh discriminant (Kloosterziel \& van Heijst 1991) and $\Omega_{b}$ is the angular velocity of the frame of reference. Thus, we now have $B_{0}=\left(S^{2}+\phi_{g}\right) /\left(S^{2}+N^{2}\right)$. Following the same reasoning as in $\S 3.2$ leads to the same quantization condition as (3.15), i.e. $\dagger$

$$
k \int_{r_{1}}^{r_{2}} \sqrt{-\frac{S^{2}+\phi_{g}}{S^{2}+N^{2}}} \mathrm{~d} u=n \pi+\frac{\pi}{2} .
$$

Using the quadratic approximation and expanding the growth rate in powers of $1 / k$ as in (3.17),

yields

$$
\sigma=\sigma_{0}+\frac{\sigma_{1}}{k}+\cdots
$$

$$
\begin{aligned}
& \sigma_{0}=-\mathrm{i} m \Omega\left(r_{0}\right)+\sqrt{-\phi_{g}\left(r_{0}\right)}, \\
& \sigma_{1}=-\frac{2 n+1}{2 \sqrt{2}} \sqrt{\phi_{g}^{\prime \prime}\left(r_{0}\right)-2 m^{2} \Omega^{\prime}\left(r_{0}\right)^{2}+2 \mathrm{i} m \sqrt{-\phi_{g}\left(r_{0}\right)} \Omega^{\prime \prime}\left(r_{0}\right)} \sqrt{1-\frac{N^{2}}{\phi_{g}\left(r_{0}\right)}},
\end{aligned}
$$

$\dagger$ The hypothesis that the turning point $r_{1}$ is not too close to the vortex axis might not be valid for some marginal values of $\Omega_{b}$. These particular cases are outside the scope of the present paper. 
where $r_{0}$ is given by

$$
\phi_{g}^{\prime}\left(r_{0}\right)=-2 \mathrm{i} m \Omega^{\prime}\left(r_{0}\right) \sqrt{-\phi_{g}\left(r_{0}\right)}
$$

The first term $\sigma_{0}$ and the equation giving $r_{0}$ are identical to (3.18) and (3.20) respectively, except that $\phi$ is replaced by the generalized Rayleigh discriminant $\phi_{g}$. The only difference shows up in the last term of (6.5) where the Brunt-Väisälä frequency appears. Therefore, the generalized Rayleigh criterion for non-axisymmetric centrifugal instabilities is modified only by the background rotation through the generalized Rayleigh discriminant $\phi_{g}$. The stratification plays a role only in the growth rate dependence on the axial wavenumber $k$.

Figure 4 compares the different asymptotic growth rates to the exact growth rates for the Carton \& McWilliams vortex for two examples: a stratified fluid $N=0.5$ and a rotating fluid $\Omega_{b}=-0.1$. For the axisymmetric mode $m=0$ (figure $4 a$ ), the growth rate given by (6.2) is indistinguishable from the exact growth rate. Expression (6.3) is also in very good agreement except at low axial wavenumbers for the rotating case $\Omega_{b}=$ -0.1 . For $m=1$ (figure $4 b$ ), the agreement is satisfactory except at low wavenumbers for the stratified case $N=0.5$ : the asymptotic growth rates (6.2) and (6.3) go to zero for $k \approx 3$ instead of $k=0$. These two examples confirm that the stratification does not affect the growth rate at large axial wavenumbers whereas the background rotation does, as predicted by the asymptotic analysis. Inversely, it is interesting to see that the stratification has a significant effect at low axial wavenumbers while the background rotation tends to have no effect. This is in full agreement with the stability analysis of Smyth \& McWilliams (1998).

\section{Taylor-Couette flow}

The case of the Taylor-Couette flow between two cylinders of radii $R_{1}<R_{2}$ rotating at angular velocities $\Omega_{1}$ and $\Omega_{2}$ is peculiar because the Rayleigh discriminant, although negative for some radius, has no radius $r^{*}$ where its derivative vanishes. Therefore, a specific analysis is needed to derive the generalized criterion for this flow. We only consider here the case of an homogeneous fluid.

The angular velocity of the Couette flow is given by

$$
\Omega=E+F / r^{2}
$$

with

$$
E=\Omega_{1}\left(\mu-\eta^{2}\right) /\left(1-\eta^{2}\right), F=\Omega_{1} R_{2}^{2} \eta^{2}(1-\mu) /\left(1-\eta^{2}\right), \mu=\Omega_{2} / \Omega_{1}, \eta=R_{1} / R_{2}
$$

(Chandrasekhar 1961). The Rayleigh discriminant

$$
\phi=4 \Omega_{1}^{2} \eta^{2} \frac{(1-\mu)\left(\mu-\eta^{2}\right)}{\left(1-\eta^{2}\right)^{2}}\left(\frac{R_{2}^{2}}{r^{2}}-\beta\right),
$$

where $\beta=\left(\eta^{2}-\mu\right) /\left((1-\mu) \eta^{2}\right)$, can be negative only if $\mu<\eta^{2}$. If such a condition is satisfied, $\phi$ increases monotonically with $r$ and is negative between $r=R_{1}$ and $r=\min \left(R_{2} / \sqrt{\beta}, R_{2}\right)$. Therefore, when $m=0$, there is at most one turning point $r_{1}$ where $B_{0}=0$. When $m \neq 0$, there exist two complex turning points but to construct valid unstable eigenmodes, it is sufficient to consider only the turning point $r_{1}$ which derives continuously from the one for $m=0$ and which has a Stokes line crossing the real axis at $r=\tilde{r}_{1}$ such that $R_{1}<\tilde{r}_{1}$ (figure 5).

The matching WKB approximations are

$$
\begin{array}{rr}
\psi_{1}(r)=\left(-B_{0}(r)\right)^{-1 / 4}\left[2 C \sin \left(k \gamma(r)+\frac{\pi}{4}\right)+D \cos \left(k \gamma(r)+\frac{\pi}{4}\right)\right] & \text { for } r<\tilde{r}_{1}, \\
\psi_{2}(r)=\left(B_{0}(r)\right)^{-1 / 4}\left[C \mathrm{e}^{-k \lambda(r)}+D \mathrm{e}^{k \lambda(r)}\right] & \text { for } r>\tilde{r}_{1},
\end{array}
$$



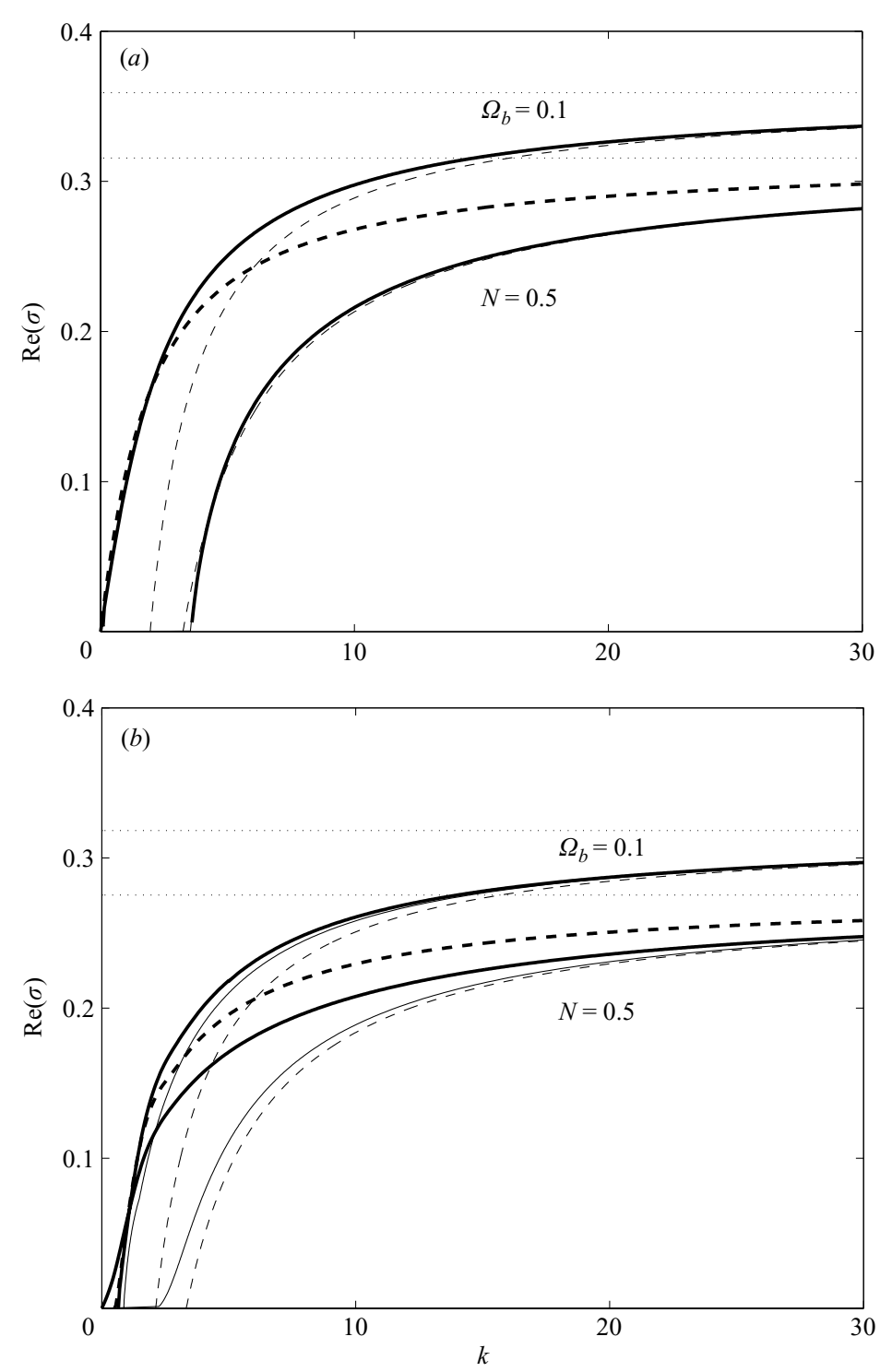

FIGURE 4. Growth rates of the azimuthal wavenumbers $(a) m=0$ and $(b) m=1$ for the Carton $\&$ McWilliams vortex for $\alpha=2$ in a stably stratified fluid $\left(N=0.5, \Omega_{b}=0\right)$ or rotating fluid $\left(N=0, \Omega_{b}=-0.1\right)$. For each case, the exact growth rate is represented by a heavy solid line and the asymptotic growth rates are shown by:,$-(6.2) ;---,(6.3) ; \cdots,(6.4)$. For reference, the exact growth rate in the homogeneous case $\left(N=0, \Omega_{b}=0\right)$ is also shown by a heavy dashed line.

where

$$
\gamma(r)=\int_{r}^{r_{1}} \sqrt{-B_{0}(u)} \mathrm{d} u, \quad \lambda(r)=\int_{r_{1}}^{r} \sqrt{B_{0}(u)} \mathrm{d} u
$$

and $(C, D)$ are constants.

When the axial wavenumber $k$ is not large and $\mu \gtrsim 0$, it is possible that the Stokes line crosses the real axis outside the outer cylinder, i.e. $\tilde{r}_{1}>R_{2}$. The WKB approximation (7.2) is then valid throughout the interval $R_{1}<r<R_{2}$. Therefore, the boundary conditions are $\psi_{1}\left(R_{1}\right)=\psi_{1}\left(R_{2}\right)=0$ imposing the condition $k \int_{R_{1}}^{R_{2}} \sqrt{-B_{0}(u)} \mathrm{d} u=\pi+n \pi$. 


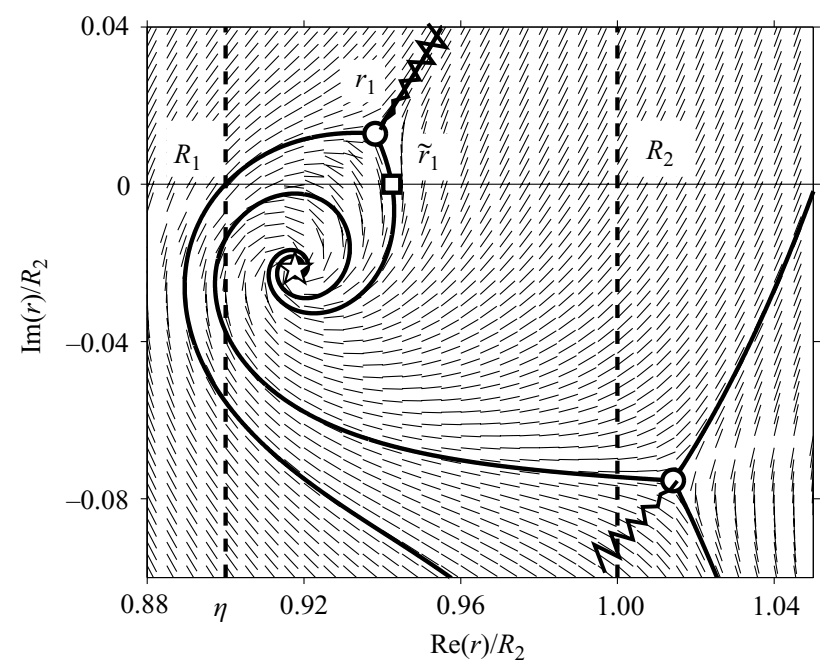

Figure 5. Example of the Stokes lines (shown by heavy lines) network for the Couette flow for $\eta=0.9, \mu=-1, m=4$ and $\sigma / \sqrt{-\phi\left(R_{1}\right)}=0.3086-0.3767$ i. Circles are turning points, the square indicates $\tilde{r}_{1}$ and the star is a singular point where $S=0$; short and wavy lines and are as in figure 2.

It can be checked that this particular condition agrees with the results of Krueger \& DiPrima (1962) for $\mu \rightarrow 1$.

However, we are interested here in the case $k \gg 1$ for which $\tilde{r}_{1}<R_{2}$. The boundary conditions are thus $\psi_{1}\left(R_{1}\right)=0$ and $\psi_{2}\left(R_{2}\right)=0$, imposing the condition

$$
\tan \left(k \gamma\left(R_{1}\right)+\pi / 4\right)=\mathrm{e}^{-2 k \lambda\left(R_{2}\right)} / 2 \text {, }
$$

i.e. at leading order in $k$

$$
k \gamma\left(R_{1}\right)=k \int_{R_{1}}^{r_{1}} \sqrt{-B_{0}(u)} \mathrm{d} u=n \pi+\frac{3 \pi}{4}+O\left(\frac{1}{k}\right),
$$

where $n$ is a non-negative integer. This condition imposes $\operatorname{Im}\left(\gamma\left(R_{1}\right)\right)=0$, implying that the points $R_{1}$ and $r_{1}$ are connected by a Stokes line (figure 5). When $k$ is large, the condition (7.4) will be satisfied only if $r_{1}$ is close to $R_{1}$ so that $B_{0}$ can be approximated by its Taylor expansion around $R_{1}: B_{0}(r)=B_{0}\left(R_{1}\right)+\left(r-R_{1}\right) B_{0}^{\prime}\left(R_{1}\right)+O\left(\left(r-R_{1}\right)^{2}\right)$. Upon integration, this leads to

$$
2 k \frac{\left(-B_{0}\left(R_{1}\right)\right)^{3 / 2}}{3 B_{0}^{\prime}\left(R_{1}\right)}=n \pi+\frac{3 \pi}{4} .
$$

For $m=0,(7.5)$ can be rewritten

$$
\left(\frac{\tilde{k} \tilde{\sigma}^{2}}{1-\mu}\right)^{2 / 3}\left[1-\frac{1}{\tilde{\sigma}^{2}}\right]=-(3 \pi(4 n+3) / 8)^{2 / 3},
$$

where $\tilde{\sigma}=\sigma / \sqrt{-\phi\left(R_{1}\right)}$ and $\tilde{k}=k R_{1}\left(1-\eta^{2}\right) / 2$. This result is very close to the small-gap formula for $k \gg 1$ (Drazin \& Reid 1981, § 16) if one notes that $\tilde{a}_{n}=-(3 \pi(4 n+3) / 8)^{2 / 3}$ approximate the zeros $a_{n}$ of the Airy function $\mathrm{Ai}$ of the small-gap formula: for $n=0$, we have $\tilde{a}_{0}=-2.320$ instead of $a_{0}=-2.338$, for $n=1, \tilde{a}_{1}=-4.081$ instead of $a_{1}=-4.088$, etc. For $m=0$ and finite values of $k$, figure 6 shows that (7.4) provides an extraordinarily accurate approximation of the numerical results, better than the small-gap formula. For small $m$ and $k \gg 1$, it can be verified that (7.5) also fully agrees with the asymptotic study of Bisshopp (1963). For finite values of $m$, the agreement 


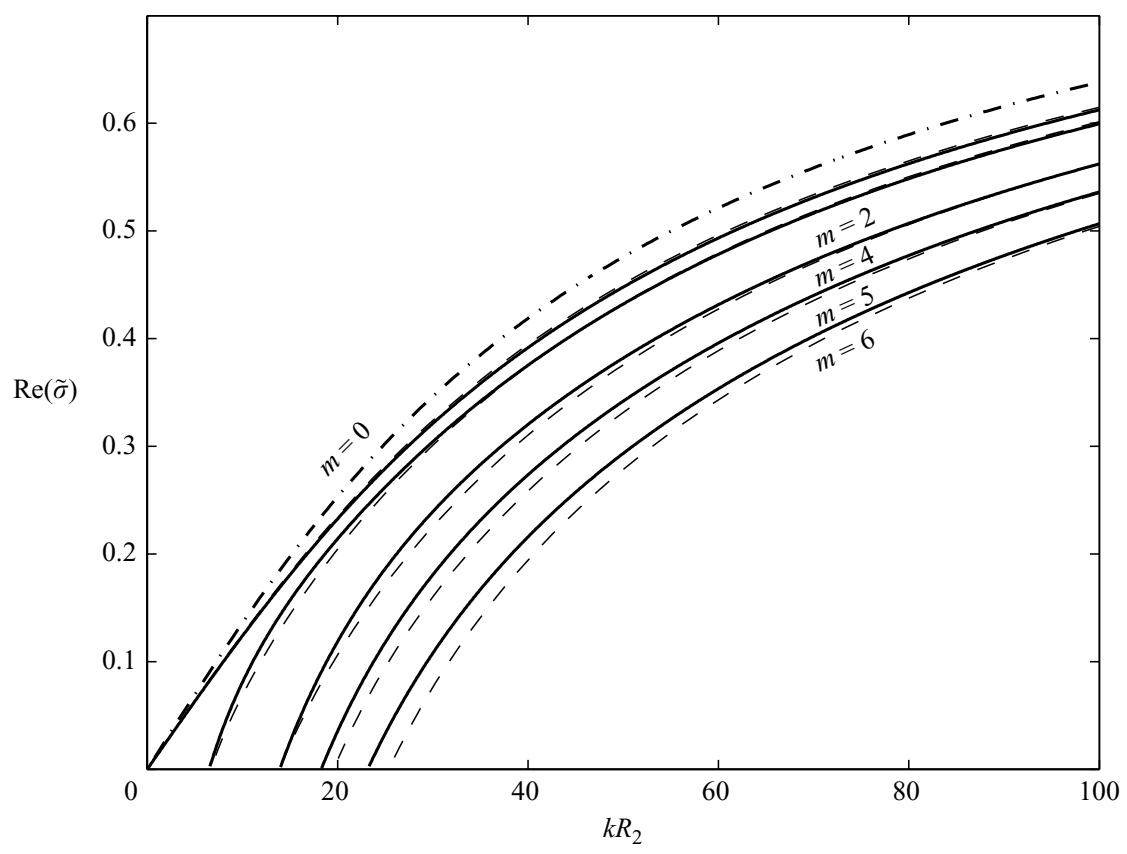

FIGURE 6. Normalized growth rates $\operatorname{Re}(\tilde{\sigma})=\operatorname{Re}(\sigma) / \sqrt{-\phi\left(R_{1}\right)}$ of the $m=(0,2,4,5,6)$ azimuthal wavenumbers for the Couette flow for $\eta=0.9, \mu=-1:-$, exact results obtained numerically; ---, (7.4);---, small-gap formula for $m=0$.

between (7.4) and the exact growth rates is excellent but tends to degrade at low $k$ as $m$ increases (figure 6).

For large axial wavenumber, the growth rate can be obtained explicitly as

$$
\sigma=\sqrt{-\phi}-\mathrm{i} m \Omega-\frac{1}{8 k^{2 / 3} \sqrt{-\phi}}\left[3 \pi(4 n+3)\left(\phi^{\prime} \sqrt{-\phi}-2 \mathrm{i} m \Omega^{\prime} \phi\right)\right]^{2 / 3}+\cdots,
$$

where all the quantities are evaluated at $r=R_{1}$. The maximum growth rate is therefore given by $\sqrt{-\phi\left(R_{1}\right)}$, which is just the minimum of $\phi$ reached at the inner cylinder boundary $r=R_{1}$. This is analogous to the case of free vortices (§3) even though the derivative of $\phi$ never vanishes. However, an important difference is that (7.7) also implies that the maximum growth rate is the same for all $m$, in sharp contrast with the results of $\S \S 3$ and 4 .

The leading-order dependence of the growth rate on the axial wavenumber is of the form $k^{-2 / 3}$ instead of $k^{-1}$ for free vortices, as recently obtained by Leblanc \& Le Duc (2005) with a different approach.

\section{Conclusions}

In this paper, we have generalized the Rayleigh criterion for centrifugal axisymmetric instabilities to non-axisymmetric perturbations by using WKB approximations for large axial wavenumber.

For unbounded vortices, the maximum growth rate of a perturbation with an azimuthal wavenumber $m$ has been found to be $\sigma_{0}=-\mathrm{i} m \Omega\left(r_{0}\right)+\sqrt{-\phi\left(r_{0}\right)}$ where $r_{0}$ is a complex radius given by $\phi^{\prime}\left(r_{0}\right)=-2 \mathrm{i} m \Omega^{\prime}\left(r_{0}\right) \sqrt{-\phi\left(r_{0}\right)}$. A sufficient condition for instability is therefore that the real part of $\sigma_{0}$ is positive. This generalized criterion also applies to a vertical vortex in a stably stratified and rotating fluid except that $\phi$ should be replaced by the generalized Rayleigh discriminant. The criterion is not valid for any complex radius $r_{0}$ satisfying the above condition. It is further required 
that two Stokes lines emanating from $r_{0}$ cross the real axis and that the WKB approximations are uniformly valid for real radius.

In the case of the Carton \& McWilliams vortices, the generalized criterion shows that non-axisymmetric perturbations are less unstable than the axisymmetric one and, more surprisingly, that they are stable above a relatively low cutoff azimuthal wavenumber. A numerical stability analysis fully confirms this result and shows a remarkable agreement between the asymptotic and exact growth rates even at low axial wavenumbers except for the azimuthal wavenumbers unstable with respect to the two-dimensional shear instability.

For the specific case of the Taylor-Couette flow, the same WKB approach shows that the maximum growth rate of non-axisymmetric perturbations is independent of $m$ because the radius $r_{0}$ is real and equal to the inner cylinder radius $R_{1}$. For finite axial wavenumber, the asymptotic growth rate also gives excellent predictions without requiring the small-gap approximation.

Further extensions to swirling jets are in progress.

We are indebted to Stéphane Le Dizès for fruitful discussions and for providing us with a draft of his paper with Laurent Lacaze while this work was in progress. We thank Colm Caulfield for careful reading of the manuscript.

\section{REFERENCES}

BAYLY, B. J. 1988 Three-dimensional centrifugal-type instabilities in inviscid two-dimensional flows. Phys. Fluids 31, 56-64.

Bender, C. M. \& Orszag, S. A. 1978 Advanced Mathematical Methods for Scientists and Engineers. McGraw-Hill.

Bisshopp, F. E. 1963 Asymmetric inviscid modes of instability in Couette flow. Phys. Fluids 6, 212-217.

Carnevale, G. F. \& Kloosterziel, R. C. 1994 Emergence and evolution of triangular vortices. $J$. Fluid Mech. 259, 305-331.

Carton, X. J. \& McWilliams, J. C. 1989 Barotropic and baroclinic instabilities of axisymmetric vortices in a quasi-geostrophic model. In Mesoscale/Synoptic Coherent Structures in Geophysical Turbulence (ed. J. Nihoul \& B. Jamart), pp. 225-244. Elsevier.

Chandrasekhar, S. 1961 Hydrodynamic and Hydromagnetic Stability. Clarendon.

Drazin, P. G. \& ReID, W. H. 1981 Hydrodynamic Stability. Cambridge University Press.

Gallaire, F. \& Chomaz, J.-M. 2003 Three-dimensional instability of isolated vortices. Phys. Fluids 15, 2113-2126.

Gent, P. R. \& McWilliams, J. C. 1986 The instability of barotropic circular vortices. Geophys. Astrophys. Fluid Dyn. 35, 209-233.

Howard, L. N. 1962 Review on hydrodynamic and hydromagnetic stability. J. Fluid Mech. 13, $158-160$.

Howard, L. N. \& GuPTA, A. S. 1962 On the hydrodynamic and hydromagnetic stability of swirling flows. J. Fluid Mech. 14, 463-476.

Kloosterziel, R. C. \& van Heisst, G. J. F. 1991 An experimental study of unstable barotropic vortices in a rotating fluid. J. Fluid Mech. 223, 1-24.

Krueger, E. R. \& DiPrima, R. C. 1962 Stability of nonrotationally symmetric disturbances for inviscid flow between rotating cylinders. Phys. Fluids 5, 1362-1367.

Leblanc, S. \& Le Duc, A. 2005 The unstable spectrum of swirling gas flows. J. Fluid Mech. 537, 433-442.

Le Dizès, S. \& LaCAZE, L. 2005 An asymptotic description of vortex Kelvin modes. J. Fluid Mech. 542, 69-96.

Olver, F. W. J. 1974 Asymptotics and Special Functions. Academic.

RaYleigh, Lord 1917 On the dynamics of revolving fluids. Proc. R. Soc. Lond. A 93, 148-154.

Smyth, W. D. \& McWilliams, J. C. 1998 Instability of an axisymmetric vortex in a stably stratified, rotating environment. Theor. Comput. Fluid Dyn. 11, 305-322.

Synge, J. L. 1933 The stability of heterogeneous liquids. Trans. R. Soc. Can. 27, 1-18. 